\title{
MÁS ALLÁ DE LAS FRONTERAS
}

Jorge Granja E. ${ }^{1}$

\section{RESUMEN}

El presente trabajo pretende dar una visión sobre la dimensión que la Educación Global tiene en el mundo contemporáneo y su alcance a nivel internacional. La tendencia que están teniendo los estudiantes jóvenes por un aprendizaje global tratando de optar por una internalización o ciudadanía global. Se concluye con resultados de una investigación que ayuda a entender por qué esta educación está siendo aceptada y promocionada por los países desarrollados.

Palabras clave: educación global, internalización, aprendizaje global, perspectiva global

\section{ABSTRACT}

The present work tries to provide a vision of the dimension that Global Education is taking in the contemporary world and its potential scope of international education. The trend that young students are taking for global learning is trying to opt for an internalization or global citizenship. It was concluded with results of a research that helps to understand why this education is being accepted and promoted by developed countries.

Key words: global education, internalization, global learning, global perspective

\section{INTRODUCCIÓN}

La Educación Global, en la actualidad, se ha convertido en un tema de mucho interés para los estudiantes jóvenes, porque esta les ofrece oportu- nidades que les permite insertarse en un mundo intercultural. El crecimiento para esta perspectiva global se atribuye a su enfoque de dar u optar por una in-

\footnotetext{
1 Pontificia Universidad Católica del Ecuador, Facultad de Comunicación, Lingüística y Literatura, Quito, Ecuador (jorgevge@hotmail.com).
} 
ternalización o ciudadanía global que les permita crecer y enrolarse con el resto del mundo y a la vez ser parte de las amenazas y conflictos que el planetay sus habitantes enfrentan. Larry Braskamp describe la internalización, término utilizado en 1980, como una "comunicación intercultural" en donde los estudiantes puedan aprender a pensar y actuar con quienes son diferentes, refiriéndose a los distintos aspectos de cultura, hábitos, creencias religiosas, etc. Sin embargo el concepto más reciente acerca de la internalización el cual ha permitido la creación de programas in- ternacionales y ha alentado a crear los últimos programas on line que están ofreciendo muchas universidades internacionales está dado por Jane Knight quién define la internalización como "process of integrating an international dimension into the teaching/ learning, research, and service functions of the university or college. An international dimension means a perspective, activity or service which introduces or integrates an international/ intercultural/global outlook into the major functions of an institution of higher education" (Knight, 2015)2.

\section{EL APRENDIZAJE GLOBAL}

Una de la investigaciones basada en niños determina que la creatividad propia de un niño para construir su propio lenguaje no se basa solo en la imitación de los sonidos o modelos que tiene a su rededor. Hoy en día, el concepto de adquisición de una nueva lengua o cualquier otro conocimiento debe ser visto desde una perspectiva global. Por ejemplo, la teoría de Noam Chomsky explica que los niños están dotados con un aparato natural para la adquisición de la lengua, este aparato se activa por ciertas experiencias lingüísticas y el crecimiento de la misma depende en gran parte de la influencia del medio en el cual se desenvuelve o crece. Esto implica que los estudiantes deben ser provistos no solo de habilidades cognitivas sino también de habilidades sociales y proporcionar el medio adecuado para su desarrollo. Chomsky da una visión en la que se involucra al individuo más allá de lo aprendido, para ser parte de algo más.

¿Por qué utilizar o considerar contenidos que surgen de la problemática global? ¿Por qué deben los educandos relacionarse con este tipo de contenido? Aparte que el hemisferio derecho de

\footnotetext{
(jorgevge@hotmail.com)

2"el proceso de integración de una dimensión internacional en la enseñanza-aprendizaje, investigación y funciones de servicio de la Universidad o el colegio. Una dimensión internacional significa una perspectiva, actividad o servicio la cual introduce o integra una representación internacional- intercultural
} 
nuestro cerebro es "global", y adquiere información de aspectos globales tanto concretos como abstractos, muchos de los planes y programas de la nueva educación en países desarrollados están considerando la incorporación de la diversidad en lengua y cultura que existe en el mundo. Por ejemplo, profesionales de la educación de instituciones con altos estándares en educación en los Estados Unidos han creado una serie de rúbricas (conjunto de criterios y estándares, generalmente relacionados con objetivos de aprendizaje, que se utilizan para evaluar un nivel de desempeño o una tarea). Una que llama la atención es la que está relacionada con el aprendizaje global; esta evalúa la interdependencia natural, cultural, económica y política de los estudiantes.

Kitty Johnson vocera oficial de la Embajada Americana, Quito, PUCE (2008), manifiesta que si incorporamos en los planes de clase y currículos, aspectos como simulación de resolución de problemas, comités de trabajo sobre proyectos reales y aprendizaje por descubrimiento, estaríamos involucrando en el sistema educativo a un mayor número de estudiantes con distintos estilos de aprendizaje. Una de las ventajas de este tipo de currículos tendría un impacto positivo en la enseñanza de los conceptos, impartiéndolos con mayor claridad e inspirando a los estudiantes a indagar más allá de sus propias fronteras y fomentar una inclusión y cooperación positiva con otras culturas.

Ernesto Santos (2007), en su tesis basada en "Las percepciones que los educadores de lenguas tienen acerca de la Educación Global" demuestra que tanto los docentes como los estudiantes concuerdan en que los problemas globales deberían ser incluidos en los contenidos a ser enseñados. Además, su estudio argumenta que la inserción de temas globales contribuiría a expandir el conocimiento de la diversidad humana y así lograr que los estudiantes tengan un acercamiento a los problemas del mundo junto al compromiso de velar por sus soluciones. La inserción de los problemas del planeta en temas de estudio abre la puerta para conectar dominios cognitivos, afectivos y participativos junto con la capacidad de ser parte de una solución.

¿Por qué los estudiantes deben aprender sobre los problemas y desafíos que son causados por aspectos globales? Existen varias tendencias a lo global que las nuevas generaciones están considerando. Una de ellas es la posibilidad de educarse fuera de los países de origen, tendencia que ha sido percibida por los jóvenes y en la cual la tecnología ha sido la mejor herramienta para que la lengua y la cultura no sean un impedimento para desarrollarse más allá de las propias fronteras. Una segunda tenden- 
cia que está tomando fuerza es la necesidad de pertenencia. El pertenecer y ser aceptado ha dado lugar al aprendizaje de nuevos idiomas dando sentido a que la migración se convierta hoy en una necesidad por la búsqueda de pertenencia y la noción de igualdad.

\section{TENDENCIA DE LA EDUCACIÓN INTERNACIONAL}

En las últimas décadas ha existido un movimiento global de estudiantes que son auspiciados por sus gobiernos para estudiar en el extranjero. Muchos países a nivel mundial han sido beneficiados por este fenómeno migratorio estudiantil. Asia, Estados Unidos y Europa han atraído a miles de estudiantes a sus universidades basados en una idea global. Así lo demuestran los registros de suscripción de estudiantes extranjeros, en donde podemos apreciar que en el caso de Asia creció en un porcentaje de 133\%, de 214.744 a 500.947, en Europa fue del $121 \%$, de 920.140 a 2.033,082 y en los Estados Unidos fue del $60 \%$, de 569.640 a 913.464 estudiantes (OECD, 2013a; p169). Cabe mencionar que para el año 2025, el número de estudiantes preparándose en universidades extranjeras se incrementará de 4.6 a 8 millones. (Goddard, 2012; OECD, 2009).

En una investigación llevada a cabo en una institución educativa en la ciudad de Quito se compararon dos tipos de contenidos. La hipótesis consistía en probar que los contenidos globales en la enseñanza del idioma Inglés, en séptimo grado de educación básica son más efectivos y significativos que los contenidos enseñados tradicionalmente y que por consiguiente son asimilados de mejor manera. Por contenidos tradicionales entendemos los que están relacionados con la moda, el espectáculo y la farándula, por contenidos globales se entienden todos aquellos que son causados por el ser humano y que tienen incidencia en los aspectos social y ambiental.

Esta investigación expone algunas razones que ayudan a entender por qué la enseñanza de contenidos globales parece ser más atractiva y por qué las instituciones educativas internacionales están proponiendo perspectivas globales a más de enseñar contenidos tradicionales. Las razones son presentadas en el orden en el cual fueron impartidas a los estudiantes en la clase. Se debe aclarar que aun cuando estamos hablando de contenidos para los niños, este tipo de contenidos resultaron interesantes para la edad de un séptimo grado. Sin embargo, los mismos temas se pueden presentar para estudiantes de un nivel universitario ya que el problema es el 
mismo. Se detectó que los estudiantes cuando utilizan contenidos globales desarrollan estrategias de curiosidad y se empoderan de un sentido de responsabilidad frente al problema e intentan, de alguna manera, buscar una solución.

\section{CONCLUSIÓN}

De la investigación realizada se pudo llegar a comprobar que los contenidos que surgen de los problemas globales se convierten en más que una simple actividad en la clase, se pudo observar la participación grupal así como la creación de posibles soluciones. Estos contenidos fomentan la participación y la disminución del temor en algunos niños ya que los contenidos trabajados eran cercanos a su realidad como ¿Quién no ha comido un hot dog? El vocabulario, aun cuando fue en inglés, dio mayor facilidad para trabajar en un contexto más amplio. Los proyectos presentados a los estudiantes permitieron dar experiencia y habilidades sociales y cognitivas para involucrarse en los problemas de su comunidad. Se percibió que existe una afinidad entre los contenidos globales, la ciencia y los estudios sociales.
La dirección que está tomando la educación por causa de la influencia tecnológica y ambiental impulsa a esta, a anclarse en una perspectiva global para enfrentar los retos de una globalización que no solamente abarca problemas ambientales sino que está involucrando a la lengua, cultura, y a la manera de sentir y pensar. Probablemente los hechos globales cambien de manera drástica la manera de vivir en el planeta y modifiquen el proceso de enseñar y aprender en el futuro, pero es claro que de alguna manera la internalización está enfocándose en aspectos que involucren a áreas lingüísticas, psicológicas, filosóficas, científicas y naturales como un solo cuerpo. 


\section{BIBLIOGRAFÍA}

Anderson, Tim (2015). Seeking Internationalization, Canadian Journal of Higher Education.

Whitehead, Michele (2015). Global Learning, Liberal Education, Vol. 101 issue3, p6-13.

Anderson, Charles (1995). Language Test Construction and Evaluation, Cambridge University Press.

Anderson, Gregory G. (1996). Global Issue in the University ESL Clasroom. Kansai Gaidai University. Recuperado /http://www.jalt-publications .org/.

Applebee (1974). The Basis of the particular needs manifested by the class.

Bartelson (2000). Globalization has destabilized the foundations of the nature of being as a new object of thought and action.

Cates, Kip A. (1991). Recuperado /http: //www.jalt-publications.org/

Cromer (1974). Relaltionships between cognitive factors and language.

Drayton, George. Education is only worth it makes in the activities of the individual who has been educated.
Freeman (1989). Applied linguistics and methodology, should not be the primary subjects matter of Language Teacher Education.

Hanvey, Robert (1976). An Attainable Global Perspective, the American Forum for Global Education. New York.

Kniep, W. (1985). A Critical Review of the Short History of Global Education. New York.

Littlewood, William T. (1984). Foreign and Second Language Teaching. Cambridge University.

Pike and Selby (1988). Phenomenon that is affecting the lives of people and of the planet.

Ramírez, Franking (1979). The most important thing in education is providing students with attitudes and capacities to solve world problems.

Santos, Ernesto (2007). Perceptions of Language Educators about Global Education...

Smith Glenda and Mike (1990). A Study Skills Handbook. Oxford University Press. 
ReVista PUCE, ISSN 1012-389X. NúM. 102

Wen-chung, Liu (2006). Memorization and Improvisation: a Comparison of Two Strategies in the Oral Acquisition of English as a Second Language.

Widdonson (1976). Foreign Language can be associated with other subjects.

Van, Ek and Alexander (1975). Whole con- text and learning and teaching and the need to consider societal and learner's needs.

Yakovchuk, Nadezhda (2001). Global Issues and Values in Foreign Language Education. Belarus. 